\title{
Analytic Solution for Fluid Flow over an Exponentially Stretching Porous Sheet with Surface Heat Flux in Porous Medium by Means of Homotopy Analysis Method
}

\author{
Azhar Ali ${ }^{*}$, H. Zaman', M. Z. Abidin'², Naeemullah', S. I. A. Shah' ${ }^{1}$ \\ ${ }^{1}$ Faculty of Numerical Science, Islamia College University, Peshawar, Pakistan \\ ${ }^{2}$ Faculty of Mechanical Engineering, CECOS University of IT and Emerging Science, Peshawar, Pakistan \\ Email: azhar ali017@yahoo.com
}

Received 23 April 2015; accepted 27 June 2015; published 30 June 2015

Copyright (C) 2015 by authors and Scientific Research Publishing Inc.

This work is licensed under the Creative Commons Attribution International License (CC BY).

http://creativecommons.org/licenses/by/4.0/

c) (i)

\begin{abstract}
In this paper, the analytical solution of a viscous and incompressible fluid towards an exponentially stretching porous sheet with surface heat flux in porous medium, for the boundary layer and heat transfer flow, is presented. The equations of continuity, momentum and the energy are transformed into non-linear ordinary differential by using similarity transformation. The solutions of these highly non-linear ordinary differential equations are found analytically by means of Homotopy Analysis Method (HAM). The result obtained by HAM is compared with numerical results presented in the literature. The accuracy of the HAM is indicated by close agreement of the two sets of results. By this method, an expression is obtained which is admissible for all values of effective parameters. This method has the ability to control the convergence of the solution.
\end{abstract}

\section{Keywords}

Exponentially Stretching Sheet, Suction/Blowing, Variable Surface Heat Flux, Porous Medium, Analytical Solution, Homotopy Analysis Method

\section{Introduction}

In fluid mechanics and heat transfer many engineering problems are basically nonlinear. The majority of these problems do not have analytical solutions. Using numerical techniques, some of them can be solved and some can be solved analytically. Stability and convergence should be considered so that the divergence can be avoided

${ }^{*}$ Corresponding author.

How to cite this paper: Ali, A., Zaman, H., Abidin, M.Z., Naeemullah and Shah, S.I.A. (2015) Analytic Solution for Fluid Flow over an Exponentially Stretching Porous Sheet with Surface Heat Flux in Porous Medium by Means of Homotopy Analysis Method. American Journal of Computational Mathematics, 5, 224-238. http://dx.doi.org/10.4236/ajcm.2015.52019 
in results obtained by numerical method. To find the analytic solution of these non linear equations, we need other methods such as perturbation method [1]. But in perturbation method, a small parameter is needed that is to be inserted in the equation. The main deficiency of perturbation method is to find that small parameter which is exerted it into the equation.

1992 [2] [3], Liao introduced the homotopy analysis method (HAM) which doesn’t need such small/large parameter and then he further improved and developed this method. The most significant feature of this method is that region of convergence can be controlled and adjusted, in comparison to other methods. It should be necessary to mention that the Homotopy Perturbation Method (HPM) brought forth in 1998, is only a particular case of HAM [4] [5].

HAM based on introduction of homotopy in topology combined with the traditional perturbation method for the solution of non linear equations, but contrary to the traditional perturbation methods, HAM doesn't need a small perturbation parameter in the equation. A homotopy is constructed with an embedding parameter p. Values are given to $\mathrm{p}$ from zero to one. The problem under consideration takes a convenient simple form which presents a closed form of analytical solution as $p=0$. Similarly if the value of $p$ increases, that finally takes the value one. Then at this stage, the solution of the original problem is obtained. The reliability of HAM also depends on two other auxiliary parameters, first is the parameter $\hbar$ and the other one is function. The choice of that function is to be practiced with in order to find out the optimal solution. With the help of $\hbar$-curve, the convergence of the analytic solution is ensured. HAM is a novel technique [6] [7], which has been used by many researchers for solving non linear ordinary differential equation. Recently, HAM has applied by many researchers to find the solution of different problems in science and engineering. Ayub [8] has considered the problem of steady, third grade fluid flowing past an infinite porous plate and for exact analytical solution of the governing non-linear differential equation, he uses HAM. On the basis of HAM, Wang and Pop [9] also proposed exact analytic solutions for flow within a non-Newtonian fluid film whose motion is caused solely by the unsteady stretching of a horizontal elastic surface. Wang [10] applied HAM to find out the explicit analytic solution of the Volterra equation. The comparison of HAM and HPM through a linear partial differential equation has been made by Liang and Jeffrey [11]. To numerically approximate the Eigen values of the fractional Sturm-Liouvile problems, Abbasbandy and Shirzadi [12] used HAM. The application of HAM was considered by Nassar [13], as he suggested that by using HAM the solution of the nonlinear Poisson-Boltzmann equation for semiconductor devices was extremely good analytical approximations. Zaman [14] studied the series solution of stagnation point flow with mass transfer along an accelerated vertical porous plate with suction by means of HAM and he found out an exact analytical solution. Also Zaman, H. [15] proposed exact analytic series solution for heat transfer from a continuous surface in a parallel free stream of viscoelastic fluid. The above discussion shows that HAM is more flexible, valid and effective for the solution of non linear ordinary differential equations arises in science and engineering.

In the present study, we use the homotopy analysis method for the solution of two non linear ordinary differential equations introduced by Mandal [16]. The distribution of the paper is as follows. In Section 2, the methematical formulation of the problem is presented. The basic idea of HAM and solution by means of HAM is discussed in Section 3. The convergence of the obtained series solutions is carefully analyzed in Section 4. The graphical results, table and discusion are presented in Section 5. The conclution is presented in Section 6.

\section{Mathematical Formulation}

\subsection{Flow Problem}

Assume the two-dimensional, steady and incompressible flow of a viscous fluid past a flat sheet coincided by the plane $y=0$ in a porous medium with a non-uniform permeability $K_{1}$. Mandal [16] considered the flow in the porous medium. The governing equation of the problem is based on Darcy's law. The Darcy's law accounts for the drag applied by the porous medium [17] [18]. There is an increase in the form drag [19] due to the significant inertial effects at maximum velocities. The inertial effects and the effects of solid bodies have been ignored. Near the boundary and in a media with high porosity [20] [21] these effects are more important. In addition, the non-linear Forhheimer term is ignored however the linear Darcy term is retained. That Darcy term describes the distributed body force exerts by porous medium. The Reynolds number was assumed to be very small in this study (typically < 10) [20] [22].

We consider Cartesian coordinates $(x ; y ; z)$. It is supposed that the sheet is associated to a variable heat 
flux $\left(\operatorname{VHWq}_{w}(x)\right)$. The flow is limited to $y>0$. Two equal and opposite forces are applied along $x$-axis, as a result the wall is stretched keeping the origin fixed. The effect of these forces causes a symmetric boundary at the centre (the origin as shown in the Figure 1) of the porous medium.

For two-dimensional flow, the velocity field is considered as

$$
V=[u(x, y), v(x, y), 0]
$$

where $u$ and $v$ are the velocity components in $x$ and $y$ directions respectively. The governing equations of continuity [23], momentum [19] [24] and energy [23] [25] are

$$
\begin{gathered}
\frac{\partial u}{\partial x}+\frac{\partial v}{\partial y}=0 \\
u \frac{\partial u}{\partial x}+\frac{\partial u}{\partial y}=v \frac{\partial^{2} u}{\partial y^{2}}-\frac{v}{k} u \\
u \frac{\partial T}{\partial x}+v \frac{\partial T}{\partial y}=\frac{\kappa}{\rho c_{p}} \frac{\partial^{2} T}{\partial y^{2}}
\end{gathered}
$$

where $\rho$ represents the fluid density (assumed constant), $v=\frac{\mu}{\rho}$ is the kinematic viscosity, $c_{p}$ is the specific heat, $\mu$ is the coefficient of fluid viscosity, $\kappa$ is the thermal conductivity.

\subsection{Boundary Conditions}

The appropriate boundary conditions for the problem are

$$
\begin{gathered}
\text { aty }=0, u=U, v=-V(x), \frac{\partial T}{\partial y}=-\frac{q_{w}(x)}{\kappa} \\
\text { asy } \rightarrow \infty, u \rightarrow 0, T \rightarrow 0
\end{gathered}
$$

Here, $U=U_{0} \mathrm{e}^{\frac{N x}{L}}$ is the stretching velocity [26], $q_{w}(x)=q_{w o} T_{O} \sqrt{\frac{U_{O}}{2 v L}} \mathrm{e}^{\frac{N x}{L}}$ is the variable surface heat flux [27], $U_{0}$ is the reference velocity, $q_{w o}$ is the heat flux and $T_{O}$ is the temperature.

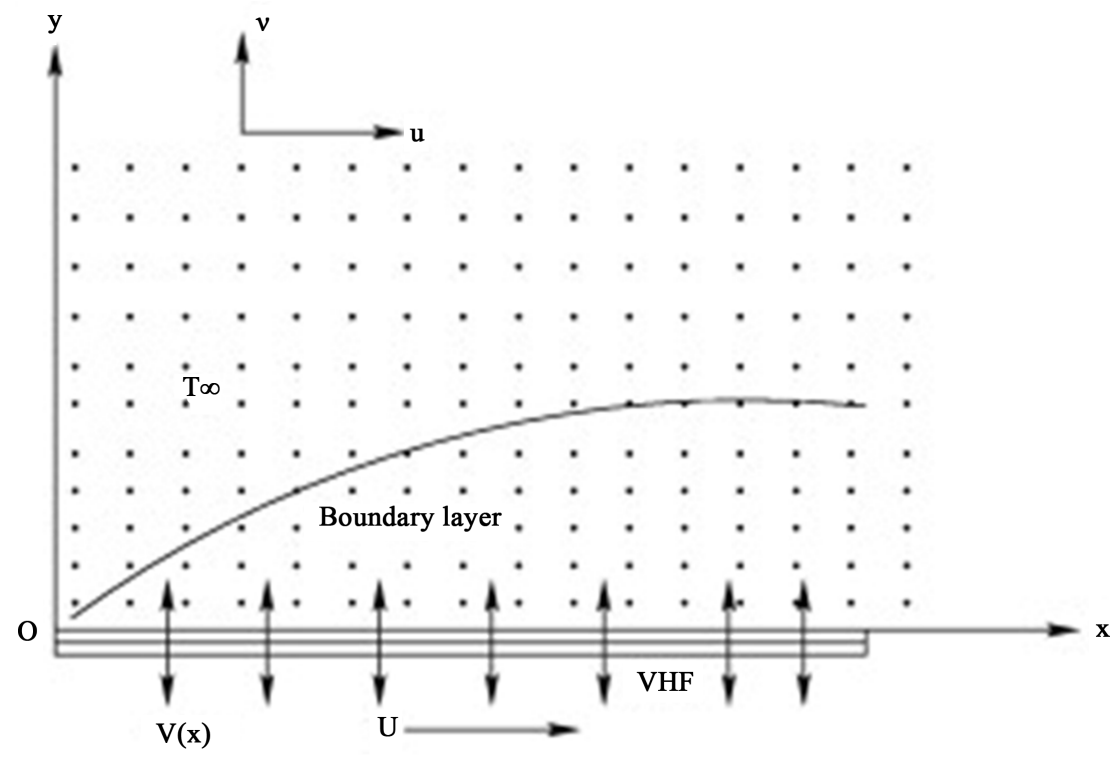

Figure 1. Geometrical representation of the problem. 
$V(x)=V_{0} \mathrm{e}^{\frac{N x}{L}}$ is a particular type of velocity at the wall is assumed [28]. where $V_{0}$ is constant, $V(x)<0$ is the velocity of blowing and $V(x)>0$ is the velocity of suction. $k=k_{0} \mathrm{e}^{\frac{N x}{L}}$ is the non-uniform permeability of the medium. Where $k_{0}$ is a constant, which gives the initial permeability and $\mathbf{N}$ is the exponential parameter.

However, for the sake of comparison, we shall consider the same case as discuss by Mandal [16] of prescribed surface temperature (PST), $T=T_{w}$ at $y=0$.

Introducing the similarity variable as

$$
\begin{gathered}
\eta=\sqrt{\frac{U_{O}}{2 v L}} \mathrm{e}^{\frac{N x}{L}} y \\
u=U_{0} \mathrm{e}^{\frac{N x}{L}} f^{\prime}(\eta) \\
v=-N \sqrt{\frac{v U_{O}}{2 L}} \mathrm{e}^{\frac{N x}{L}}\left\{f(\eta)+\eta f^{\prime}(\eta)\right\} \\
T=\frac{q_{w o}}{\kappa} T_{O} \mathrm{e}^{\frac{N x}{L}} \theta(\eta)
\end{gathered}
$$

and by substituting (7a)-(7d) in Equations (3) and (4), we obtain

$$
\begin{gathered}
f^{\prime \prime}+N f f^{\prime}-2 N f^{\prime 2}-K_{1} f^{\prime}=0 \\
\theta^{\prime \prime}+\operatorname{Pr}\left(N f \theta^{\prime}-N f^{\prime} \theta\right)=0
\end{gathered}
$$

And the boundary conditions become as:

$$
\begin{aligned}
& \text { at } \eta=0, f^{\prime}=1, f=S, \theta^{\prime}=-1 \\
& \text { and as } \eta \rightarrow \infty, f^{\prime} \rightarrow 0, \theta \rightarrow 0
\end{aligned}
$$

where the prime represents differentiation with respect to $\eta, S=\frac{V_{O}}{\sqrt{\frac{\nu U_{O}}{2 L}}}<0($ or $>0)$ is the blowing (or suction) parameter, $\operatorname{Pr}=\frac{\mu c_{p}}{\kappa}$ is the Prandtl number and $K_{1}=\frac{2 v L}{\kappa_{O} U_{O}}$ is the permeability parameter.

\section{Homotopy Analysis Method}

\subsection{Basic Idea}

Assume the following non-linear differential equation in the form of

$$
A[g(t)]=0
$$

where $A$ is a non-linear operator and $g(t)$ is the solution of equation. By defining the function $\varphi(t, p)$ such as

$$
\lim _{p \rightarrow 0} \varphi(t, p)=g_{o(t)}
$$

where $p \epsilon[0,1]$ and $g_{o(t)}$ is the initial approximation that satisfy initial or boundary conditions and

$$
\lim _{p \rightarrow 1} \varphi(t, p)=g_{(t)}
$$

Then by applying the generalized homotopy method, known as zero-order deformation Equation (12) is

$$
(1-p) \mathcal{L}\left[\varphi(t, p)-g_{o}(t)\right]=p \hbar \mathcal{H}(t) A[t, p]
$$


where $\hbar$ is the auxiliary parameter called control parameter. $\mathcal{H}(t)$ is the auxiliary function, $\mathcal{L}$ is the linear operator. It is noticed that there is a great independency for choosing the initial guess $g_{o}(t)$, the auxiliary linear operator $\mathcal{L}$, the auxiliary parameter $\hbar$ and the auxiliary function $\mathcal{H}(t)$. The discussed independency has a significant role in the strong flexibility and validity of HAM as presented in this paper.

So, as $\mathrm{p}$ takes values from 0 to 1 , the solution $\varphi(t, p)$ changes among the initial guess $g_{o}(t)$ to the solution $g(t)$. For $\varphi(t, p)$, the Taylor's series expansion with respect to $\mathrm{p}$ is given by

$$
\varphi(t, p)=g_{o(t)}+\sum_{m=1}^{\infty} g_{m}(t) p^{m}
$$

and

$$
g_{o}^{[m]}(t)=\left.\frac{\partial^{m} \varphi(t, p)}{\partial p^{m}}\right|_{p=0}
$$

where $g_{o}^{[m]}$ is known as the $m$ th order of deformation, that leads to

$$
g_{m}(t)=\frac{g_{o}^{[m]}}{m \cdot !}=\left.\frac{1}{m !} \frac{\partial^{m} \varphi(t, p)}{\partial p^{m}}\right|_{p=0}
$$

By defining the vector of

$$
\boldsymbol{g}_{\bar{m}}=\left\{g_{o}, g_{1}, g_{2}, \cdots, g_{m}\right\}
$$

By the definition in Equation (17), the governing equation and the corresponding initial conditions of $g(t)$ can be obtained from zero order deformation Equation (12). Differentiating Equation (12) $m$ th-times with respect to $p$ and considering $p=0$ and finally dividing by $m$ !, we get the $m$ th-order deformation equation in this form

$$
\mathcal{L}\left[g_{m}(t)-\chi_{m} g_{m-1}(t)\right]=\hbar \mathcal{H}(t) R\left(g_{m-1}\right)
$$

where

$$
R\left(\boldsymbol{g}_{\bar{m}-1}\right)=\left.\frac{1}{(m-1) !} \frac{\partial^{m-1} A[\varphi(t, p)]}{\partial p^{m-1}}\right|_{p=0}
$$

And

$$
\chi_{m}= \begin{cases}0, & m \leq 1, \\ 1, & m>1\end{cases}
$$

As we apply inverse operator $\mathcal{L}^{-1}$ to both sides of the Equation (20), we get

$$
g_{m}(t)=\chi_{m} g_{m-1}(t)+\hbar \mathcal{L}^{-1} \mathcal{H}(t) R\left(g_{m-1}\right)
$$

In this way, we can easily solve the Equation (12) to obtained $g_{m}$ for $m \geq 1$ at $m$ th-order, we have

$$
g(t)=\sum_{m=1}^{M} g_{m}(t)
$$

By using the initial or boundary conditions we find the constant(s).

As $M \rightarrow+\infty$, we obtain a precise approximation of the Equation (12). The convergence of the above method is discussed in detail by Liao [2]. If Equation (12) has a unique solution, then HAM will produce the unique solution, otherwise the HAM will produce a solution between many other (possible) solutions.

\subsection{The Solution of the Problem by Means of HAM}

\subsubsection{Zero-Order Deformation Problem}

To find the series solution, $f(\eta)$ and $\theta(\eta)$ can be written by the set of base functions

$$
\left\{\eta^{n}, n \geq 0\right\}
$$




$$
\begin{aligned}
& f(\eta)=\sum_{n=0}^{\infty} a_{n} \eta^{n} \\
& \theta(\eta)=\sum_{n=0}^{\infty} b_{n} \eta^{n}
\end{aligned}
$$

where $a_{n, n}$ and $b_{n, n}$ are coefficients.

For the problem under discussion, the initial guesses and the auxiliary linear operators has to be chosen as

$$
\begin{gathered}
f_{o}(\eta)=(S+1)-\mathrm{e}^{-\eta} \\
\theta_{O}(\eta)=\mathrm{e}^{-\eta} \\
\mathcal{L}_{1}(f(\eta))=\frac{\mathrm{d}^{3} f}{\mathrm{~d} \eta^{3}}+\frac{\mathrm{d}^{2} f}{\mathrm{~d} f^{2}} \\
\mathcal{L}_{2}(\theta(\eta))=\frac{\mathrm{d}^{2} \theta}{\mathrm{d} \eta^{2}}+\frac{\mathrm{d} \theta}{\mathrm{d} \eta}
\end{gathered}
$$

With

$$
\begin{gathered}
\mathcal{L}_{1}\left(C_{1}+C_{2} \eta+C_{3} \mathrm{e}^{-\eta}\right)=0 \\
\mathcal{L}_{2}\left(C_{4}++C_{5} \mathrm{e}^{-\eta}\right)=0
\end{gathered}
$$

And $C_{i}(i=1, \cdots, 5)$ are the orbitrary constants.

For the probem, the zero-order deformation is

$$
\begin{gathered}
(1-p) \mathcal{L}_{1}\left[\varphi(t, p)-f_{o}(t)\right]=p \hbar_{1} A_{1}[\varphi(t, p)] \\
\varphi(0, p)=S,\left.\frac{\partial \varphi(\eta, p)}{\partial \eta}\right|_{\eta=0}=1 \\
\left.\frac{\partial \varphi(\eta, p)}{\partial \eta}\right|_{\eta=\infty}=0 \\
(1-p) \mathcal{L}_{2}\left[\psi(t, p)-\theta_{o}(t)\right]=p \hbar_{2} A_{2}[\varphi(t, p), \psi(t, p)] \\
\psi(\infty, p)=0,\left.\frac{\partial \psi(\eta, p)}{\partial \eta}\right|_{\eta=0}=-1 \\
A_{1}[\varphi(t, p)]=\frac{\partial^{3} \varphi(\eta, p)}{\partial \eta^{3}}+N \varphi(\eta, p) \frac{\partial^{2} \varphi(\eta, p)}{\partial \eta^{2}}-2 N\left(\frac{\partial \varphi(\eta, p)}{\partial \eta}\right)^{2}-K_{1} \frac{\partial \varphi(\eta, p)}{\partial \eta} \\
A_{2}[\varphi(t, p), \psi(t, p)]=\frac{\partial^{2} \psi(\eta, p)}{\partial \eta^{2}}+\operatorname{Pr}\left(N \varphi(t, p) \frac{\partial \psi(\eta, p)}{\partial \eta}-N \frac{\partial \varphi(\eta, p)}{\partial \eta} \psi(t, p)\right)
\end{gathered}
$$

where $\left(p \int[0,1]\right)$ and $\hbar_{i} \neq(i=1,2)$ are the respective embedding and auxiliary parameter such that $\phi(\eta, 0)=f_{o}(\eta), \phi(\eta, 1)=f(\eta)$ and $\psi(\eta, 0)=\theta_{0}(\eta), \psi(\eta, 1)=\theta(\eta)$. Obviouslywhen $\mathrm{p}$ varies from 0 to 1 , $\phi(\eta, p)$ changes from the initial guess $f_{o}(\eta)$ to exact solution $f(\eta)$ and $\psi(\eta, p)$ from $\theta_{0}(\eta)$ to $\theta(\eta)$. By Taylor's series, we have

$$
\begin{gathered}
\phi(\tau, p)=f_{o}(\tau)+\sum_{m=1}^{\infty} f_{m}(\tau) p^{m} \\
\psi(\tau, p)=\theta_{o}(\tau)+\sum_{m=1}^{\infty} \theta_{m}(\tau) p^{m}
\end{gathered}
$$




$$
\begin{aligned}
& f_{m}(\tau)=\left.\frac{1}{m !} \frac{\partial^{m} \phi(\tau, p)}{\partial p^{m}}\right|_{p=0,} \\
& \theta_{m}(\tau)=\left.\frac{1}{m !} \frac{\partial^{m} \psi(\tau, p)}{\partial p^{m}}\right|_{p=0,}
\end{aligned}
$$

\subsubsection{Higher-Order Deformation Problem}

The $m$ th-order deformation problem are

$$
\begin{gathered}
\mathcal{L}_{1}\left[f_{m}(\tau)-\chi_{m} f_{m-1}(\tau)\right]=\hbar_{1} R_{1 m}\left(f_{m-1}\right) \\
f_{m}^{\prime}(0)=1, f_{m}^{\prime}(\infty)=0, f_{m}(0)=S \\
\mathcal{L}_{2}\left[\theta_{m}(\tau)-\chi_{m} \theta_{m-1}(\tau)\right]=\hbar_{2} R_{2 m}\left(f_{m-1} \theta_{m-1}\right) \\
\theta_{m}^{\prime}(0)=-1, \theta_{m}(\infty)=0
\end{gathered}
$$

where

$$
\begin{gathered}
R_{1 m}\left(f_{m-1}\right)=f_{m-1}^{\prime \prime \prime}+\sum_{n=0}^{m-1}\left[N f_{n}(\eta) f_{m-1-n}^{\prime \prime}(\eta)-2 N f_{n}^{\prime}(\eta) f_{m-1-n}^{\prime}(\eta)-K_{1} f_{m-1}^{\prime}(\eta)\right] \\
R_{2 m}\left(f_{m-1}, \theta_{m-1}\right)=\theta_{m-1}^{\prime \prime}(\eta)+\operatorname{Pr} \sum_{n=0}^{m-1}\left[N f_{n}(\eta) \theta_{m-1-n}^{\prime}(\eta)-N f_{n}^{\prime}(\eta) \theta_{m-1-n}(\eta)\right]
\end{gathered}
$$

And

$$
\chi_{m}= \begin{cases}0, & m \leq 1 \\ 1, & m>1\end{cases}
$$

The general soluton of the Equations (45)-(48) is

$$
\begin{gathered}
f_{m}(\eta)=f_{m}^{*}(\eta)+C_{1}^{m}+C_{2}^{m} \eta+C_{3}^{m} \mathrm{e}^{-\eta} \\
\theta_{m}(\eta)=\theta_{m}^{*}(\eta)+C_{4}^{m}+C_{5}^{m} \mathrm{e}^{-\eta}
\end{gathered}
$$

In which $f_{m}^{*}(\eta)$ and $\theta_{m}^{*}(\eta)$ represent the special solution of Equations (45) and (47) and the integral constants $C_{i}(i=1-5)$ can be computed by empolying the boundary conditions (46) and (48) as:

$$
\begin{gathered}
C_{1}^{m}+C_{3}^{m}=f_{m}^{*}(0), C_{2}^{m}-C_{3}^{m}=f_{m}^{* *}(0), C_{2}^{m}=f_{m}^{* *}(\infty) \\
C_{4}^{m}=\theta_{m}^{*}(\infty), C_{5}^{m}=\theta_{m}^{* *}(0)
\end{gathered}
$$

thus, it is convenient to find the solution of Equations (44)-(47), continuously in the order $m=1,2,3, \cdots$, in particular by using the symbolic computation software Mathematica.

\section{Convergence of the Series Solutions}

For HAM solution, the rate of convergence of approximation strongly depends on auxiliary parameter $\hbar$, which has big effect on the region of convergence. Due to the region of convergence and rate of convergence of a series are essentially determined by the base functions, its convergence is guaranteed.

We observe that the series solutions (24) and (25) contain the non-zero auxilary parameter $\hbar_{1}$ and $\hbar_{2}$. The suitable values for $\hbar_{1}$ and $\hbar_{2}$ can be compute by $\hbar$-curve. Figure 2(a) shows the variation of $f^{\prime \prime}(0)$ with $\hbar_{1}$ using the $12^{\text {th }}$ order of HAM approximation. Figure 2(a) describes that convergent result can be find out by taking a value of $\hbar_{1}$ from the range $[-1.5,0]$. To find the proper value for $\hbar_{1}$, we take the same case as discussed by Mandal [16] for permeability parameter $K_{1}=1$ and the three values of suction/injection parameter $S=-1,0,1$ in Figure 3(a). Also for exponentail parameter $N=1$ and for the same three values of suction/injection parameter $S=-1,0,1$ in Figure 3(b). We tried different values of $\hbar_{1}$ in the range $[-1.5,0]$ 


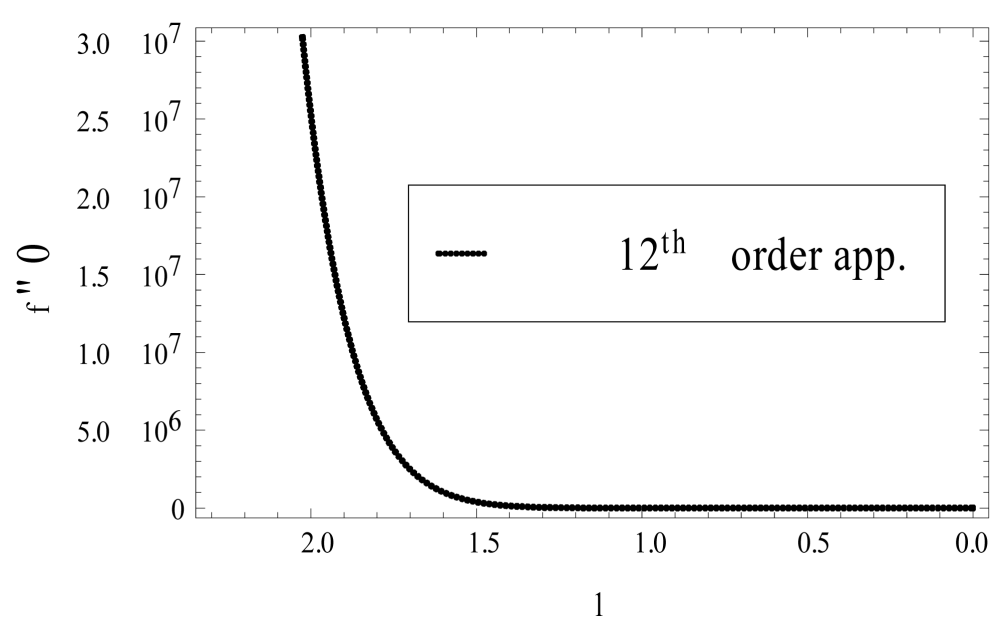

(a)

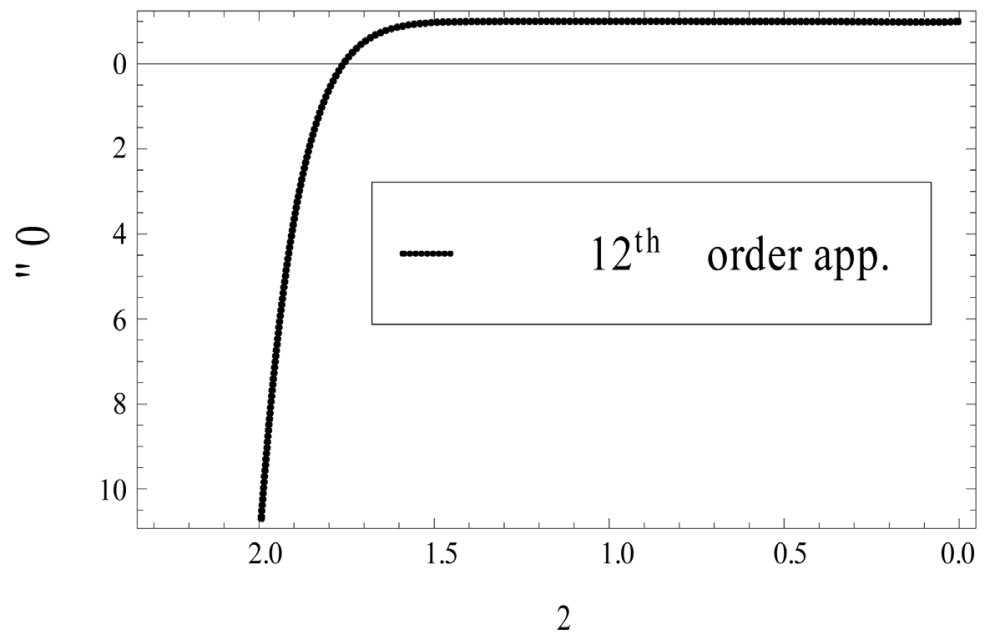

(b)

Figure 2. (a) $\hbar_{1}$-curve are plotted for $12^{\text {th }}$ order of approximation when $K_{1}=0, N$ $=1, S=0$ and $\operatorname{Pr}=1$; (b) $\hbar_{2}$-curve are plotted for $12^{\text {th }}$ order of approximation when $K_{1}=0, N=1, S=0$ and $P r=1$.

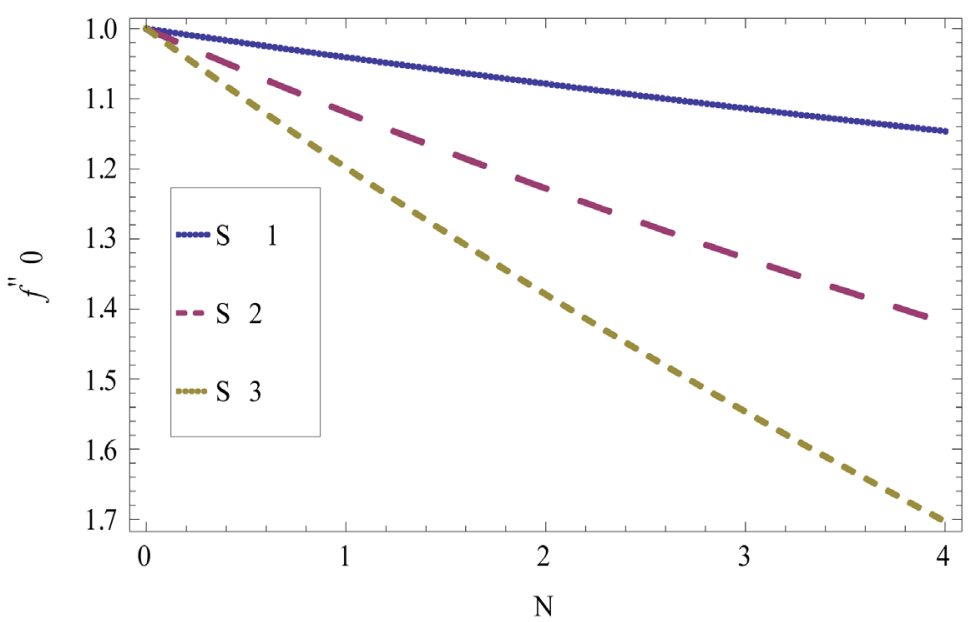

(a) 


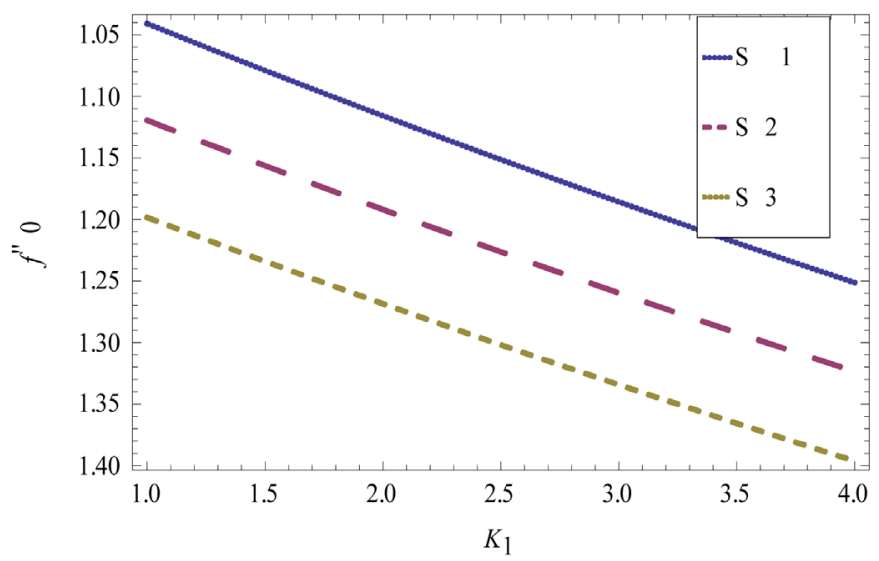

(b)

\begin{abstract}
Figure 3. (a) Skin-friction coeficient $f^{\prime \prime}(0)$ against exponential exponent $N$ for three values of suction/injection parameter $S . K_{1}=1$; (b) Skin-friction coeficient $f^{\prime \prime}(0)$ against permeability parameter $K_{1}$ for three values of suction/injection parameter $S . N=1$.
\end{abstract}

for Figure 3(a) and Figure 3(b) and compared with results obtained by Mandal [16], which shows that the best value for $\hbar_{1}$ is -0.01 . Figure 2(b). represents the range for the admisible values for $\hbar_{2}$ is also $[-1.5,0]$. For finding the suitable value of $\hbar_{2}$, we consider the same case as considered by Mandal [16] shown in Figure 4(b), Figure 5(b), Figure 6(b), Figure 6(d) and Figure 7. We tried different values of $\hbar_{2}$ in the range $[-1.5,0]$ and compared with results obtained by Mandal [16], which shows that the best value for $\hbar_{2}$ is $-0.01,-0.1$ and -0.11 .

\title{
5. Table, Graphical Results and Discussion
}

In this paper, we consider the same study as discussed by Mandal [16]. Solution of Equations (8) and (9) with the boundary conditions (10) and (11) are find out by means of HAM. For the varification of accuracy of the results obtained by HAM a comparison is made with the results, obtained by Mandal [16], Magyari and Keller [26], Bidin and Nazar [23], El-Aziz [29] and Ishak [25], for prescribed surface temperature (PST) instead of variable surface heat flux. For this comparison, we also consider the boundary conditions for temprature as follows:

At $y=0, T=T_{w}$ and as $y \rightarrow \infty, T \rightarrow 0$ where $T=T_{O} \mathrm{e}^{\frac{N x}{2 L}}$ is the temprature at the sheet.

At last, the boundary conditions take the given form:

At $\eta=0, \theta=1$ and as $\eta \rightarrow \infty, \theta \rightarrow 0$ (PST).

A comparison is made for the obtained results for precribed surface temprature (PST) coresponding to the values of heat transfer coefficient $\left[-\theta^{\prime}(0)\right]$ for different values of parandal number with $N=1, K_{1}=0, N=1$ and $S=0$ (i.e. for non-porous medium and in the absence of suction/blowing at the boundary) with the available published results of Magyari and Keller [26], Bidin and Nazar [23], El-Aziz [29], Ishak [25] and Mandal [16] are presented in Table 1 . The obtained results show an excellent agreement. These results are computed by different values of $\hbar_{1}$ and $\hbar_{2}$ from their intervals of convergence respectively, for different orders of approximations.

The analytical solution obtained by HAM has a high order of accuracy with a few iterations.

In order to analyze the effect of various parameters on the flow and temperature profile, analytical computations have been carried out for variable surface heat flux(VHF) using the HAM consider the same case as described by Mandal [16]. To see the effect of different parameters of interest on the velocity and tempprature profile, we have plotted the Figures 3(b)-7.

Let us first discuus with the existence of suction at the wall the effect of exponential parameter $N$ on velocity and temprature profile. 


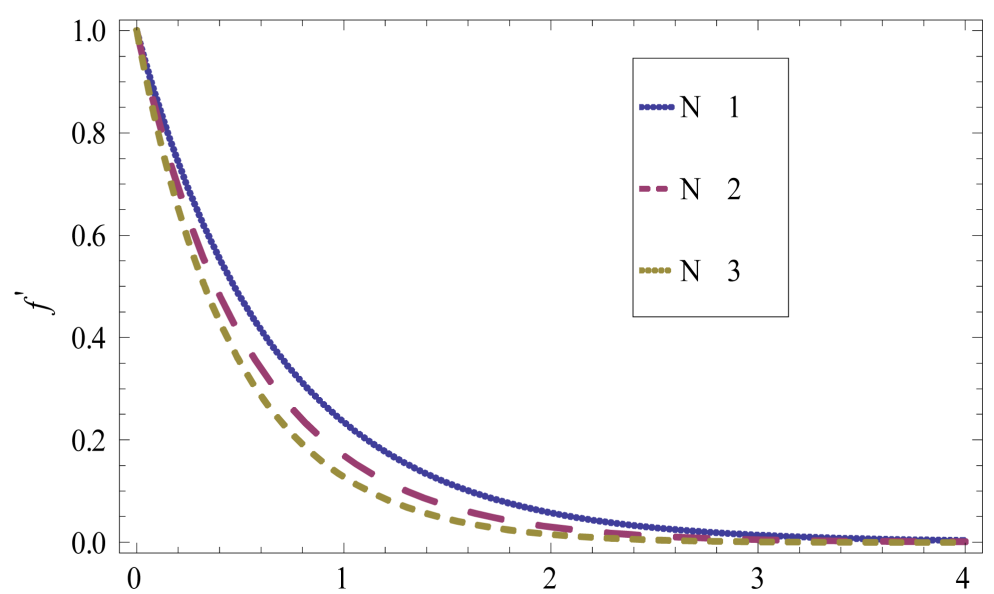

(a)

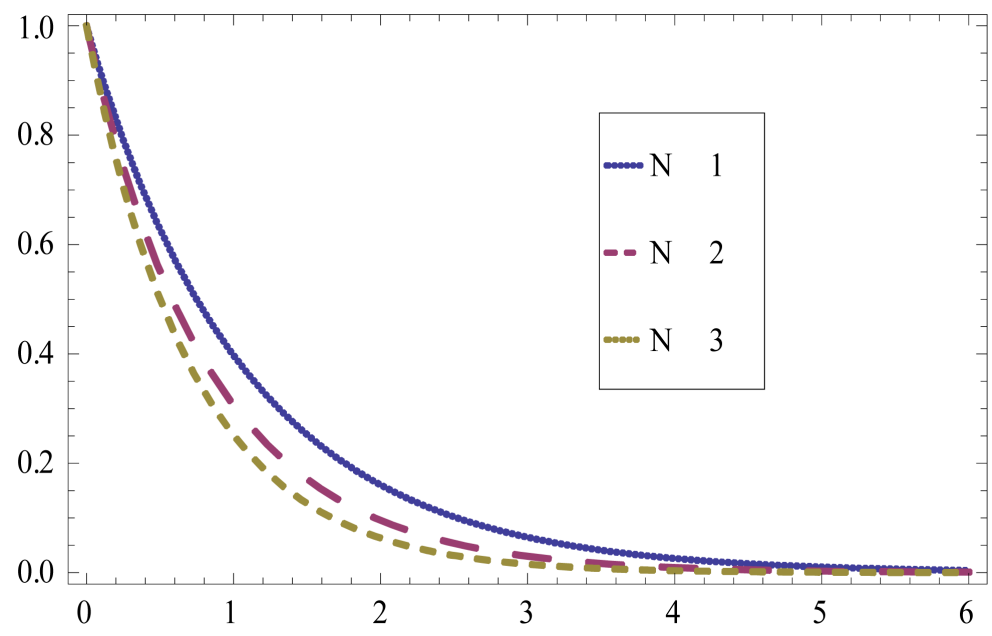

(b)

Figure 4. (a) Variation of velocity $f^{\prime}(\eta)$ with $\eta$ for different values of exponential parameter $N, K_{1}=1, S=0$; (b) Variation of temprature $\theta(\eta)$ with $\eta$ for different values of exponential parameter $N, K_{1}=1, S=0$ and $\operatorname{Pr}=1$.

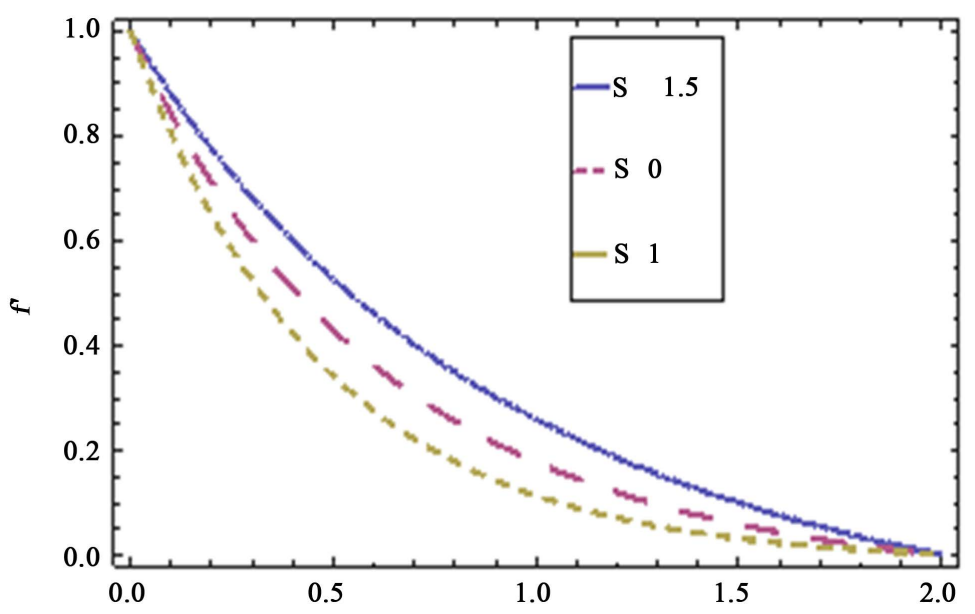

(a) 


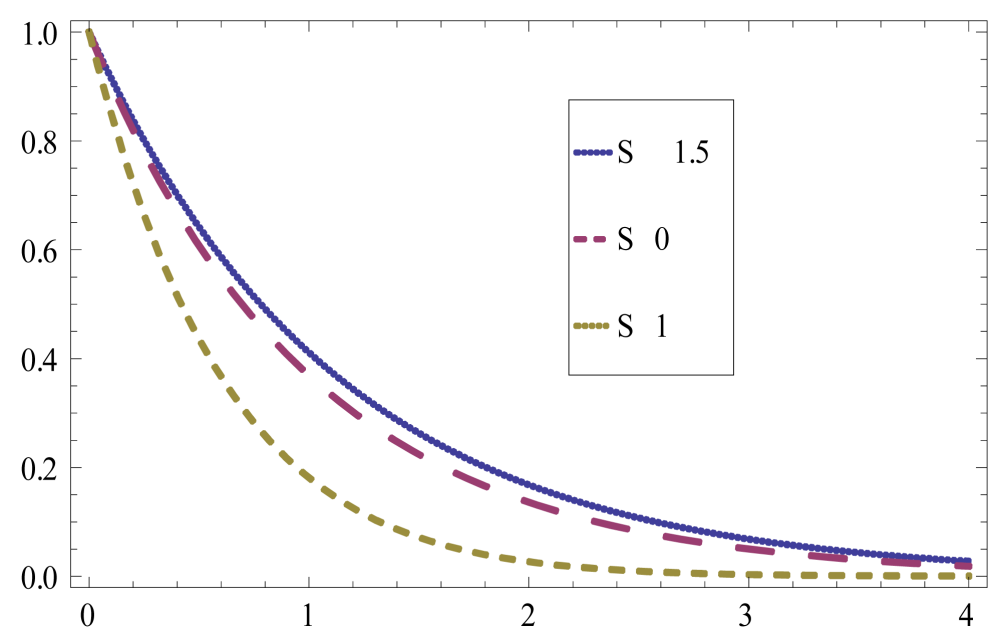

(b)

Figure 5. (a) Variation of velocity $f^{\prime}(\eta)$ with $\eta$ for different values of Suction/blowing parameter $S, K_{1}=1, N=1$; (b) Variation of velocity $\theta(\eta)$ with $\eta$ for different values of Suction/blowing parameter $S, K_{1}=1, N=1$, and $\operatorname{Pr}=1$.

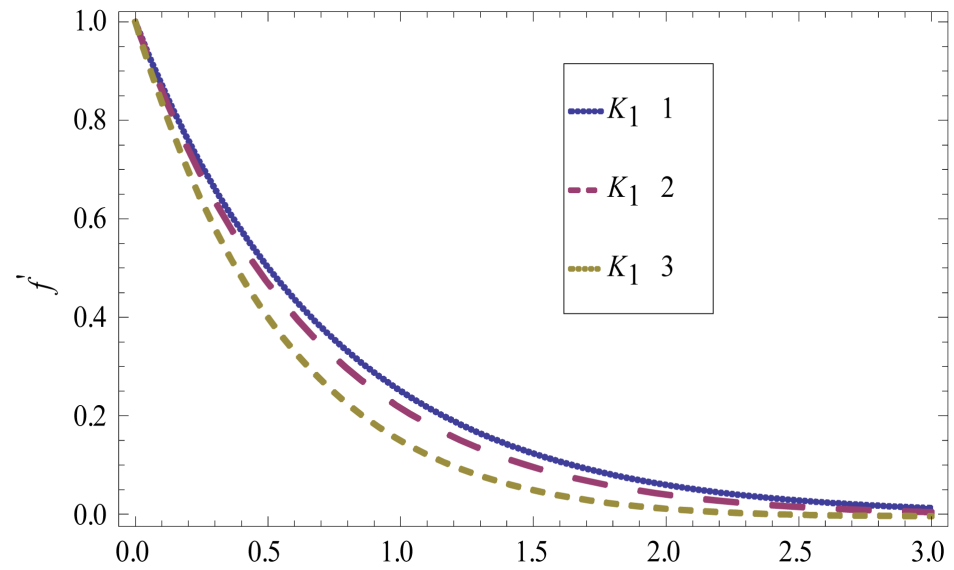

(a)

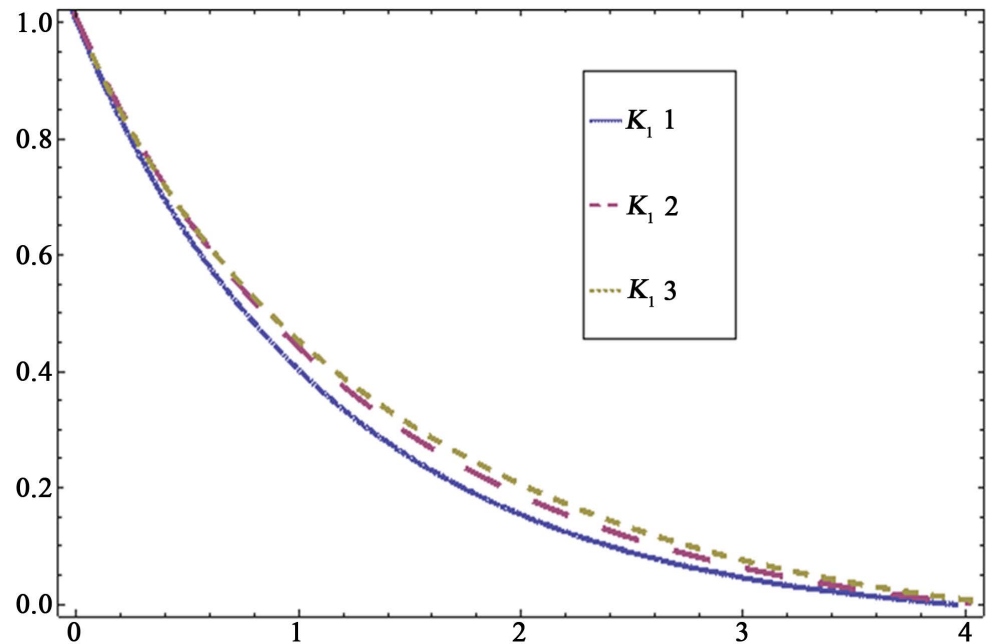

(b) 


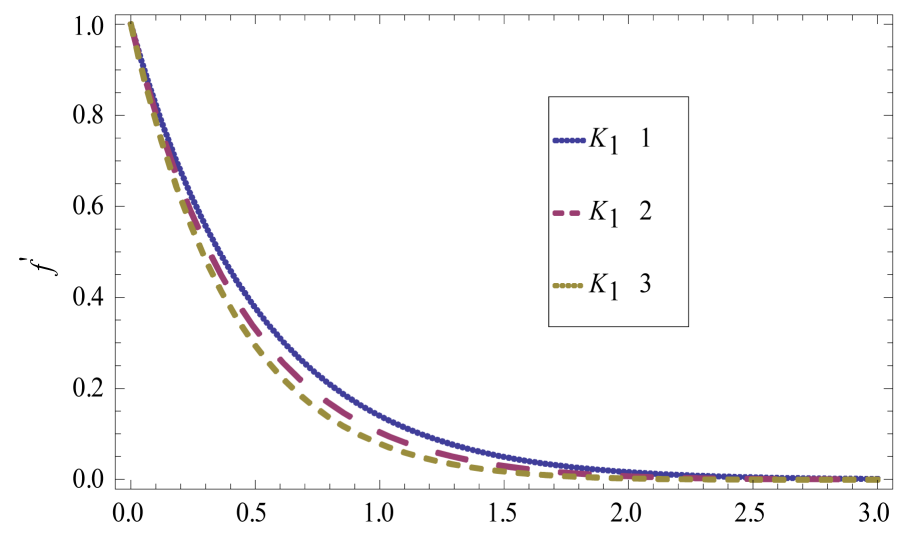

(c)

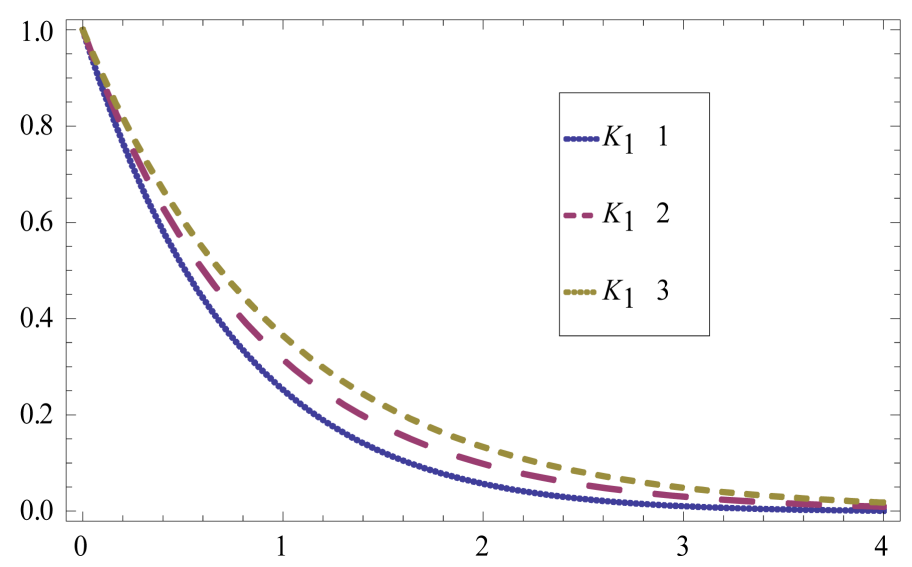

(d)

Figure 6. (a) Variation of velocity $f^{\prime}(\eta)$ with $\eta$ for different values of permeability parameter $K_{1}$ in the absent of suction. $N=1, S=0$; (b) Variation of velocity $\theta(\eta)$ with $\eta$ for differentvalues of permeability parameter $K_{1}$ in the absence of suction. $N=1, p r=1, S=1$; (c) Variation of velocity $f^{\prime}(\eta)$ with $\eta$ for different values of permeability parameter $K_{1}$ in the presence of suction. $N=1$, $S=1$; (d) Variation of temprature $\theta(\eta)$ with $\eta$ for different values of permeability parameter $K_{1}$ in the presence of suction. $\operatorname{Pr}=1, N=1, S=1$.

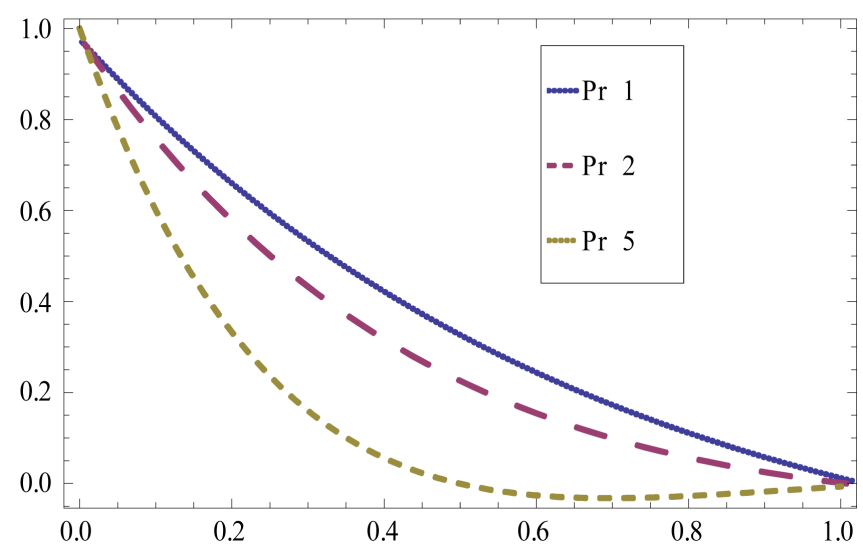

Figure 7. Variation of temprature $g(\eta)$ with $\eta$ for differentvalues of prandtl number $\operatorname{Pr} . K_{1}=1$, $N=1, S=1$. 
Table 1. Comparison of the present analytical results with available numerical results for $\left[-\theta^{\prime}(0)\right](P S T)$ for several values of Prandal number.

\begin{tabular}{ccccccccccc}
\hline Pr & $\begin{array}{c}\text { Magyari and } \\
\text { Keller [26] }\end{array}$ & $\begin{array}{c}\text { Bidin and } \\
\text { Nazar [23] }\end{array}$ & $\begin{array}{c}\text { El-Aziz } \\
{[29]}\end{array}$ & $\begin{array}{c}\text { Ishak } \\
{[25]}\end{array}$ & $\begin{array}{c}\text { Mandal } \\
{[16]}\end{array}$ & HAM & $\begin{array}{c}\text { Order of } \\
\text { approximation }\end{array}$ & $\hbar_{1}$ & $\hbar_{2}$ \\
\hline 1 & 0.9548 & 0.9547 & 0.9548 & 0.9548 & 0.9547 & 0.9547 & 14 & -0.2 & -0.2 \\
2 & & 1.4714 & & 1.4715 & 1.4714 & 1.4714 & 11 & -0.3 & -0.3 \\
3 & 1.8691 & 1.8691 & 1.8691 & 1.8691 & 1.8691 & 1.8691 & 19 & -0.4 & -0.4 \\
5 & 2.5001 & & 2.5001 & 2.5001 & 2.5001 & 2.5001 & 32 & -0.2 & -0.2 \\
10 & 3.6604 & & 3.6604 & 3.6604 & 3.6603 & 3.6607 & 23 & -0.00002 & -0.1 \\
\hline
\end{tabular}

We consider the same case as discussed by Mandal [16]. Figure 4(a) and Figure 4(b) present the effect of exponential parameter $N$ with variable surface heat flux on velocity and temprature profile. Both velocity and temprature decreases with increasing in $N$ due to decreasing behaviour of the momentum and thermal boundary layer thickness. From these figures, it is intresting to mention that the wall temprature decreses for positive value of Nthroughout in the boundary layer.

for exponentially streaching sheet, to see the effect of suction/blowing parameter $S$ on velocity and temprature profile are presented in Figure 5(a) and Figure 5(b) respectively. It is noticed that by increasing suction velocity dereases significantly whereas with increse in blowing fluid velocity is found to increase (Figure 5(a)). It is notice that for the wall suction $(S>0)$ the boundary layer thikness decreases and the velocity field is reduced. The case of non-porous sretching sheet is represent by $S=0$. Noted opposite behaviour for blowing $(S<$ 0 ). If stronger blowing is consideredthe heated fluid is pushed far from the wall where the flow is accelerated due to less influence of the viscosity. This behaviour increases maximum velocity in the boundary layer. In case of suction the same rule is working but in opposite direction. Figure 5(b) represents the temprature profile for variable suction/blowing parameter $\mathrm{S}$ with surface heat flux. By increasing suction it is observerd that decreases whereas temprature increases due to blowing (Figure 5(b)). Actually, the effect of suction more uniform within the boundary layer. At the surface, imposition of fluid suction has the tendency to reduce both the thermal thickness and hydrodynamic of the boundary layer where viscous effect domminate. Due to this effect both the fluid velocity and temnprature are reduced. Alternatively, with injection the thermal boundary layer thickness increase due to which rate of heat transfer decreases.

For the case $S=0$, the influence of permeabikity parameter $K_{1}$ on velocity and temprature are exhibited in Figure 6(a) and Figure 6(b) repectivelly. It is clear that in the presence of a porous medium the fluid flow hashigher restrictionthat, in turn, slows in motion. consequently, at the surface the shear stress increases [20]. Therefore, with increases in permiability parameter, increases the resistance to the fluid motion. With this effect the fluid velocity decreases (Figure 6(a)) and due to which in the boundary layer there is rise in temprature (Figure 6(b)) which implyes that the heat transfer rate improves by Darcian body force. It can thus be infered that an increase in permiability parameter derease the boundary layer thickniss and consequenctly increases in the rate of heat transfer.

In the presence of suction, Figure 4(c) and Figure 4(d) show the effect of the permiability parameter on the velocity and temprature profiles respectivelly. In the presnce of suction, fluid velocity is suppressed a bit more (compare to $S=0$ case) with increasing permiiability parameter $K_{1}$ (Figure 6(c)). In this case, a little bit temprature is found to increase (Figure 6(d)) due to combined effect of permiability parameter and suction.

Figure 7 presents the effect of prandtl number Pr on the temprature profile. In presence of variable heat flux, the temprature decreases with the Pr. The thermal bundary layer thicness is reduced due to increase in $\operatorname{Pr}$. In heat transfer problem, the relative thickneing of the momentum and thermul boundary layeris controlled by the prandtl number Pr. With a small Prandtl number $\mathrm{Pr}$, heat diffuses fastly compared to the velocity ( momentum), that is for liquid metals, the thickness of the boundary layer is much bigger than momentum boundary layer. That fluids whichhave higher thermal conductivity with lower Prandtl number (and thicker thermal boundary layer structures) can diffuse from the sheet faster as compared to that fluid which have higher $\operatorname{Pr}$ fluids (thinner htermal boundary layers). Thus, Prandtl increases the rate of cooling in conducting flows [20].

Figure 6(a) shows the behaviour of skin-friction coefficient with exponential parameter $N$ for three different 
values of suction/blowing parameter $S$. It is observed that skin-friction coefficient $\left[-f^{\prime \prime}(0)\right]$ increase with N.

The skin-friction coefficient it is higher for suction than that of blowing.

It is observed from = Figure 3(a) that at the wall stress is negative, negative sign of $f^{\prime \prime}(0)$ physically indicates that surface exerts a drugging force on the fluid and positive sign indicates the opposite.

Figure 3(b) diplays against the permeability parameter $K_{1}$ the nature of skin-friction coefficient. By increasing permiability parameter the Skin-friction coefficient $\left[-f^{\prime \prime}(0)\right]$ increase.Due to increase of permiability parameter $K_{1}$ the skin-friction alsoincrease. An additional shear stress on the boundary is introduced by the permeability parameter $K_{1}$.

\section{Conclusion}

In this paper, the homotopy analysis method is used to obtain the analytical solutions of a non linear Ordinary differential equations related to the boundary layer flow and heat transfer flow of a viscous and incompressible fluid towards an exponentially stretching porous sheet with surface heat flux in porous medium. The convergence of the HAM solution is discussed in detail. Definitely, the HAM gives us a simple way to control the convergence of series. That is the fundamental difference between the HAM and other analytical methods. The effect of the emerging parameters is discussed and the results are presented graphically. The results obtained by HAM are compared with the numerical results as discussed in the literature and with other stated available results. The comparision shows acceptable agreement between analytical and numerical solutions.

\section{References}

[1] He, J.-H. (2003) Homotopy Perturbation Method: A New Nonlinear Analytical Technique. Applied Mathematics and Computation, 135, 73-79. http://dx.doi.org/10.1016/S0096-3003(01)00312-5

[2] Liao, S.J. (2003) Beyond Perturbation: Introduction to the Homotopy Analysis Method. CRC Press, Boca Raton. http://dx.doi.org/10.1201/9780203491164

[3] Liao, S.J. (1992) The Proposed Homotopy Analysis Technique for the Solution of Nonlinear Problems. Ph.D. Thesis, Shanghai Jiao Tong University, Shanghai.

[4] Sajid, M., Hayat, T. and Asghar, S. (2007) Comparison between the HAM and HPM Solutions of Thin Film Flows of Non-Newtonian Fluids on a Moving Belt. Nonlinear Dynamics, 50, 27-35. http://dx.doi.org/10.1007/s11071-006-9140-y

[5] Liao, S.J. (2005) Comparison between the Homotopy Analysis Method and Homotopy Perturbation Method. Applied Mathematics and Computation, 169, 1186-1194. http://dx.doi.org/10.1016/j.amc.2004.10.058

[6] Liao, S.J. (2009) Notes on the Homotopy Analysis Method: Some Definitions and Theorems. Communications in Nonlinear Science and Numerical Simulation, 14, 983-997. http://dx.doi.org/10.1016/j.cnsns.2008.04.013

[7] Liao, S.J. (2012) Homotopy Analysis Method in Nonlinear Differential Equations. Springer, Berlin and Beijing. http://dx.doi.org/10.1007/978-3-642-25132-0

[8] Ayub, M., Rasheed, A. and Hayat, T. (2003) Exact Flow of a Third Grade Fluid Past a Porous Plate Using Homotopy Analysis Method. International Journal of Engineering Science, 41, 2091-2103. http://dx.doi.org/10.1016/S0020-7225(03)00207-6

[9] Wang, C. and Pop, I. (2006) Analysis of the Flow of a Power-Law Fluid Film on an Unsteady Stretching Surface by Means of Homotopy Analysis Method. Journal of Non-Newtonian Fluid Mechanics, 138, 161-172. http://dx.doi.org/10.1016/j.jnnfm.2006.05.011

[10] Wang, Z., Zou, L. and Zhang, H. (2007) Applying Homotopy Analysis Method for Solving Differential-Difference Equation. Physics Letters A, 369, 77-84. http://dx.doi.org/10.1016/j.physleta.2007.04.070

[11] Liang, S. and Jeffrey, D.J. (2009) Comparison of Homotopy Analysis Method and Homotopy Perturbation Method through an Evolution Equation. Communications in Nonlinear Science and Numerical Simulation, 14, 4057-4064. http://dx.doi.org/10.1016/j.cnsns.2009.02.016

[12] Abbasbandy, S. and Shirzadi, A. (2010) Homotopy Analysis Method for Multiple Solutions of the Fractional SturmLiouville Problems. Numerical Algorithms, 54, 521-532. http://dx.doi.org/10.1007/s11075-009-9351-7

[13] Nassar, C.J., Revelli, J.F. and Bowman, R.J. (2011) Application of the Homotopy Analysis Method to the PoissonBoltzmann Equation for Semiconductor Devices. Communications in Nonlinear Science and Numerical Simulation, 16, 2501-2512. http://dx.doi.org/10.1016/j.cnsns.2010.09.015 
[14] Zaman, H. and Ayub, M. (2010) Series Solution of Unsteady Free Convection Flow with Mass Transfer along an Accelerated Vertical Porous Plate with Suction. Central European Journal of Physics, 8, 931-939.

[15] Zaman, H., Hayat, T., Ayub, M. and Gorla, R.S.R. (2011) Series Solution for Heat Transfer from a Continuous Surface in a Parallel Free Stream of Viscoelastic Fluid. Numerical Methods for Partial Differential Equations, 27, 1511-1524. http://dx.doi.org/10.1002/num.20593

[16] Mandal, I.C. and Mukhopadhyay, S. (2013) Heat Transfer Analysis for Fluid Flow over an Exponentially Stretching Porous Sheet with Surface Heat Flux in Porous Medium. Ain Shams Engineering Journal, 4, 103-110. http://dx.doi.org/10.1016/j.asej.2012.06.004

[17] Pal, D. and Hiremath, P.S. (2010) Computational Modeling of Heat Transfer over an Unsteady Stretching Surface Embedded in a Porous Medium. Meccanica, 45, 415-424.

[18] Joseph, D.D., Nield, D.A. and Papanicolaou, G. (1982) Nonlinear Equation Governing Flow in a Saturated Porous Medium. Water Resources Research, 18, 1049-1052. http://dx.doi.org/10.1029/WR018i004p01049

[19] Brinkman, H.C. (1949) A Calculation of the Viscous Force Exerted by a Flowing Fluid on a Dense Swarm of Particles. Applied Scientific Research, 1, 27-34. http://dx.doi.org/10.1007/BF02120313

[20] Mukhopadhyay, S. and Layek, G.C. (2012) Effects of Variable Fluid Viscosity on Flow past a Heated Stretching Sheet Embedded in a Porous Medium in Presence of Heat Source/Sink. Meccanica, 47, 863-876. http://dx.doi.org/10.1007/s11012-011-9457-6

[21] Vafai, K. and Tien, C.L. (1981) Boundary and Inertia Effects on Flow and Heat Transfer in Porous Media. International Journal of Heat and Mass Transfer, 24, 195-203. http://dx.doi.org/10.1016/0017-9310(81)90027-2

[22] Beg, O.A., Bhargava, R., Rawat, S., Takhar, H.S. and Beg, T.A. (2007) Finite Element Modeling of Laminar Flow of a Third Grade Fluid in a Darcy-Forcheimmer Porous Medium with Suction Effects. International Journal of Applied Mechanics and Engineering, 12, 215-233.

[23] Biliana, B. and Nazar, R. (2009) Numerical Solution of the Boundary Layer Flow over an Exponentially Stretching Sheet with Thermal Radiation. European Journal of Scientific Research, 33, 710-717.

[24] Cortell, R. (2005) Flow and Heat Transfer of a Fluid through a Porous Medium over a Stretching Surface with Internal Heat Generation/Absorption and Suction/Blowing. Fluid Dynamics Research, 37, 231-245. http://dx.doi.org/10.1016/j.fluiddyn.2005.05.001

[25] Ishak, A. (2011) MHD Boundary Layer Flow Due to an Exponentially Stretching Sheet with Radiation Effect. Sains Malaysiana, 40, 391-395.

[26] Magyari, E. and Keller, B. (1999) Heat and Mass Transfer in the Boundary Layers on an Exponentially Stretching Continuous Surface. Journal of Physics D: Applied Physics, 32, 577-585. http://dx.doi.org/10.1088/0022-3727/32/5/012

[27] Nadeem, S., Zaheer, S. and Fang, T. (2011) Effects of Thermal Radiation on the Boundary Layer Flow of a Jeffrey Fluid over an Exponentially Stretching Surface. Numerical Algorithms, 57, 187-205. http://dx.doi.org/10.1007/s11075-010-9423-8

[28] Bhattacharyya, K. (2011) Boundary Layer Flow and Heat Transfer over an Exponentially Shrinking Sheet. Chinese Physics Letters, 28, Article ID: 074701. http://dx.doi.org/10.1088/0256-307X/28/7/074701

[29] Abd El-Aziz, M. (2009) Viscous Dissipation Effect on Mixed Convection Flow of a Micropolar Fluid over an Exponentially Stretching Sheet. Canadian Journal of Physics, 87, 359-368. http://dx.doi.org/10.1139/P09-047 